\title{
Minimizing Transmission Loss in Smart Microgrids by Sharing Renewable Energy
}

\author{
ZHICHUAN HUANG and TING ZHU, University of Maryland, Baltimore County \\ DAVID IRWIN, University of Massachusetts, Amherst \\ ADITYA MISHRA, Seattle University \\ DANIEL MENASCHE, Federal University of Rio de Janeiro \\ PRASHANT SHENOY, University of Massachusetts, Amherst
}

\begin{abstract}
Renewable energy (e.g., solar energy) is an attractive option to provide green energy to homes. Unfortunately, the intermittent nature of renewable energy results in a mismatch between when these sources generate energy and when homes demand it. This mismatch reduces the efficiency of using harvested energy by either (i) requiring batteries to store surplus energy, which typically incurs $\sim 20 \%$ energy conversion losses, or (ii) using net metering to transmit surplus energy via the electric grid's AC lines, which severely limits the maximum percentage of renewable penetration possible. In this article, we propose an alternative structure where nearby homes explicitly share energy with each other to balance local energy harvesting and demand in microgrids. We develop a novel energy sharing approach to determine which homes should share energy, and when to minimize system-wide energy transmission losses in the microgrid. We evaluate our approach in simulation using real traces of solar energy harvesting and home consumption data from a deployment in Amherst, MA. We show that our system (i) reduces the energy loss on the AC line by $64 \%$ without requiring large batteries, (ii) performance scales up with larger battery capacities, and (iii) is robust to different energy consumption patterns and energy prediction accuracy in the microgrid.
\end{abstract}

Categories and Subject Descriptors: J.7 [Computer Applications]: Computers in Other SystemsCommand and control

General Terms: Design, Measurement, Management

Additional Key Words and Phrases: Microgrid, renewable energy, energy sharing, battery

ACM Reference Format:

Zhichuan Huang, Ting Zhu, David Irwin, Aditya Mishra, Daniel Menasche, and Prashant Shenoy. 2016. Minimizing transmission loss in smart microgrids by sharing renewable energy. ACM Trans. Cyber-Phys. Syst. 1, 2, Article 5 (December 2016), 22 pages.

DOI: http://dx.doi.org/10.1145/2823355

This research was supported by NSF grants CNS-1503590, IIP-1534080, CNS-1405826, CNS-1253063, and the Massachusetts Department of Energy Resources. Prof. Ting Zhu is the corresponding author of this article.

Authors' addresses: Z. Huang, ITE 367, 1000 Hilltop Cir, Baltimore, MD 21250, USA; email: zhihu1@ umbc.edu; T. Zhu, ITE 360, 1000 Hilltop Cir, Baltimore, MD 21250, USA; email: zt@umbc.edu; D. Irwin, University of Massachusetts, ECE Department, 151 Holdsworth Way, 211D Knowles Engineering Building, Amherst, MA 01003, USA; email: irwin@ecs.umass.edu; A. Mishra, Engineering 507, Seattle University, Seattle, WA 98122, USA; email: mishraa@seattleu.edu; D. Menasche, Av. Brigadeiro Trompowsky s/n CT bloco H sala 319, Ilha do Fundao, Rio de Janeiro, RJ 68511, Brasil; email: danielsadoc@gmail.com; P. Shenoy, Department of Computer Science, 140 Governors Drive, University of Massachusetts Amherst, MA 01003, USA; email: shenoy@cs.umass.edu.

Permission to make digital or hard copies of part or all of this work for personal or classroom use is granted without fee provided that copies are not made or distributed for profit or commercial advantage and that copies show this notice on the first page or initial screen of a display along with the full citation. Copyrights for components of this work owned by others than ACM must be honored. Abstracting with credit is permitted. To copy otherwise, to republish, to post on servers, to redistribute to lists, or to use any component of this work in other works requires prior specific permission and/or a fee. Permissions may be requested from Publications Dept., ACM, Inc., 2 Penn Plaza, Suite 701, New York, NY 10121-0701 USA, fax +1 (212) 869-0481, or permissions@acm.org.

(c) 2016 ACM $2378-962 X / 2016 / 12-A R T 5 \$ 15.00$

DOI: http://dx.doi.org/10.1145/2823355 


\section{INTRODUCTION}

To reduce carbon footprint, energy harvesting devices (e.g., solar panels) are becoming increasingly popular in homes nowadays. With the renewable energy generated by solar panels and energy storage units (e.g., batteries), homes can be integrated as a small, independent energy community. The power systems applied in such communities are referred to as microgrids. If the harvested energy surpasses the consumption, then microgrids can work independently and autonomously.

However, the amount of renewable energy harvested normally does not match the amount of energy consumed in individual homes. One solution is to sell the surplus energy to a utility company and get energy from the utility company during energy shortages. However, a large amount of energy generated by homes can destabilize the power system if transmitted through an alternating current (AC) line. Another solution is to store the energy in local batteries. However, this approach requires extremely large size batteries to store energy for a whole day's energy consumption. In this article, we solve the mismatch of energy harvesting and consumption by introducing energy sharing among homes. If renewable energy can be shared and consumed among homes, the traditional power system will not be interrupted by the renewable energy. To realize the energy sharing, we propose a hybrid design of energy transmission system, which contains both the traditional AC line and a small community level direct current (DC) power line. Compared with a traditional AC line, our DC line is significantly shorter. Therefore, the energy transmission loss over the DC line is much lower than the loss over the traditional AC line. With energy sharing, the total amount of energy needed from the AC line is significantly reduced, which, in turn, dramatically reduced the total energy transmission loss from the AC line.

One of our aims is to keep the system compatible with a traditional AC power grid and reduce the deployment and maintenance cost. Therefore, homes are connected to a single DC power line (called DC main bus). This introduces another design challengehow to monitor and quantify the amount of energy shared among homes. If homes provide (or extract) energy to (or from) the DC power line at the same time, we cannot fully control the amount of energy shared between different pairs of homes. To address this challenge, we propose a novel energy transmission protocol for controllers to communicate with homes to avoid transmission chaos. In our approach, homes will send out energy information to controllers and controllers then decide energy sharing pairs and send out control signals to homes. During the transmission process, homes and controller will react with signals from each other to avoid transmission chaos. Moreover, our approach enables (i) multiple homes to simultaneously provide energy to a single home, and (ii) a single home to simultaneously provide energy to multiple homes. In this way, our approach not only allows us to monitor and quantify the amount of energy shared among homes but also reduces the time required for energy sharing. However, since only one transmission can be executed at one time in a cluster, it is important to decide which cluster each home belongs to. Thus, we propose a cluster selection algorithm to organize clusters with homes that have a complementary energy consumption pattern to speed up transmissions.

Since transmission loss among homes differs for every pair of homes, an efficient algorithm is needed to control the energy sharing flow. Generally, the energy loss between homes includes two parts: (i) energy loss over wires, and (ii) energy conversion loss during the battery charging and discharging stages. Energy loss over wires is mainly determined by the distance of homes. To reduce the energy transmission loss, we propose a greedy energy matching algorithm to reduce the distance of energy transmission and the amount of energy needed to be stored in the battery. Specifically, the main contributions of the article can be summarized as follows: 

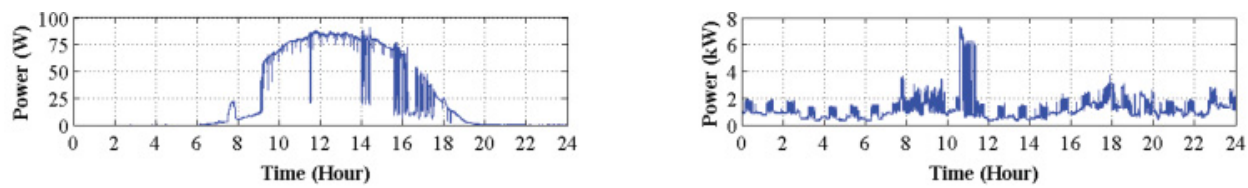

(a) Energy harvesting rate of one solar panel unit (b) Energy consumption rate of a residential over 1 day

building over 1 day

Fig. 1. Mismatching between energy harvesting and energy consumption.

- To the best of our knowledge, this is the first in-depth work to investigate the coexistence of a traditional AC power grid and a DC power line for sharing renewable energy among homes.

-We have (i) designed an efficient energy sharing system to share energy among homes, (ii) developed a lightweight energy matching algorithm and cluster selection algorithm to minimize the total energy transmission loss based on the predicted energy consumption and energy harvesting, and (iii) proposed an energy transmission protocol to monitor and quantify the amount of energy shared among homes.

-We set up a series of experiments with empirical data to verify the effectiveness of our system. The results indicate that our system can reduce $64 \%$ of energy loss from an AC line by efficiently sharing energy among homes and reduce battery size significantly. We also investigate the robustness of the system and verify that our system can work with different energy consumption patterns and energy prediction accuracy higher than $65 \%$ in the microgrid.

The article is organized as follows. In Section 2, we discuss the need for energy sharing. Section 3 gives an overview of the system architecture. Models, detailed system design, and evaluations are presented in Sections 4, 5, and 6, respectively. Practical issues and related work are discussed in Sections 7 and 8, respectively. We conclude our article in Section 9.

\section{MOTIVATION}

This work is motivated by the mismatch between energy harvesting and energy consumption in a single home, as shown in Figure 1. The mismatch occurs mainly due to the differences between the time when renewable energy is harvested and each home's peak demand time. As a result, today's Distributed Generation (DG) deployments rely heavily on net metering, where consumers sell the unused energy they produce back to the utility company. DG is a much less attractive option if net metering is not available. Net metering laws and regulations vary widely across states; it is not available in at least 4 of the United States and the regulations are weak in many others [State Environmental Resource Center 2011]. Unfortunately, even where available, states typically place caps on both the total number of participating customers and/or the total amount of energy contributed per customer [Rose and Chapman 2009]. After exceeding these caps, utilities are no longer obligated to accept excess power from DG deployments. For example, the state of Washington caps the total number of participating customers at $0.25 \%$ of all customers. One reason for the strict laws limiting DG's contribution is that injecting significant quantities of power into the grid from unpredictable energy sources at large scales has the potential to destabilize the grid by making it difficult, or impossible, for utilities to balance supply and demand.

Today's energy prices do not make DG financially attractive enough for consumers to reach even these low state caps. However, more widespread adoption of environmentally friendly renewable energy sources is critical to meet existing goals. For example, the Renewables Portfolio Standard targets 25\% of electricity generation from 


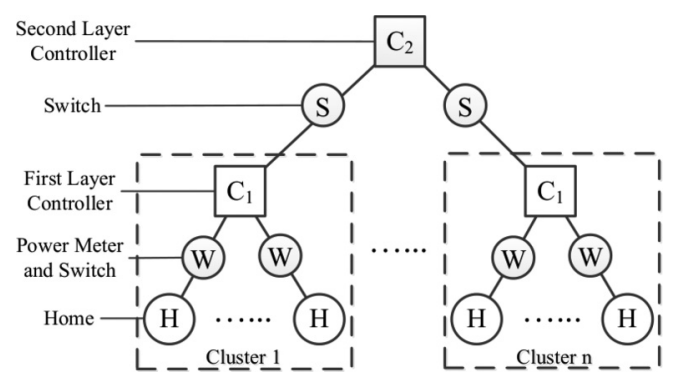

Fig. 2. Tree topology of homes.

intermittent renewables [DSIRE 2010], while California's Executive Order S-21-09 calls for 33\% of generation from renewables by 2020 [State of California 2009]. Given current laws, if DG becomes more widespread, residential consumers will have to look beyond net metering to reduce costs and balance on-site energy production and consumption. One alternative approach is to use on-site energy storage (such as batteries). However, batteries are expensive. For example, Nissan Leaf's battery price is $\$ 18,000$ for $24 \mathrm{KWh}$ [DOE 2014]. In T. Zhu et al. [2011], we have demonstrated that a $12 \mathrm{KWh}$ battery cannot buffer sufficient renewable energy for a regular home's daily energy consumption. Moreover, charging and discharging batteries introduce significant energy conversion loss. Therefore, we propose to share harvested energy among small-scale networked homes to balance the energy harvesting and consumption in a larger group (i.e., a microgrid). Since the distances between homes are typically way shorter than from homes to the utility company, energy sharing can significantly reduce energy transmission loss.

\section{SYSTEM OVERVIEW}

In this section, we briefly overview the system architecture and introduce the hardware architecture to achieve energy sharing among multiple homes.

Our energy sharing system uses a cluster controller to control the energy sharing among homes. The cluster controller collects information from homes and then arranges the transmission among homes. To guarantee backward compatibility with a traditional power system, we adopt the tree topology in Figure 2, which is similar to the one used in a traditional power grid, to connect homes for energy sharing. Homes are connected to the cluster controller with both a power meter and a switch. The power meter is used to measure energy harvesting and consumption information while the switch is used to control how to share energy with other homes. Since cluster controllers do not need to measure energy information, they are connected to higher layer controllers only through switches. In our system, energy is first shared among homes within a cluster and then shared among homes in different clusters in a higher layer repeatedly until all possible energy transmissions are finished.

To realize energy sharing, we propose a three-layer design, as shown in Figure 3.

- The hardware layer uses a power meter to measure (i) the energy harvesting rate from renewable energy devices such as solar panels; (ii) the energy consumption rate from appliances such as heating devices. This energy information will be transferred to local control layer for prediction of future energy harvesting and consumption rate periodically. The hardware layer will then execute energy transmission with the control signal transferred from power control. The proposed hardware architecture is shown in Figure 4. The harvesting devices (solar panel in our article) are directionally connected to a power meter and a switch, since the energy cannot be transmitted to 


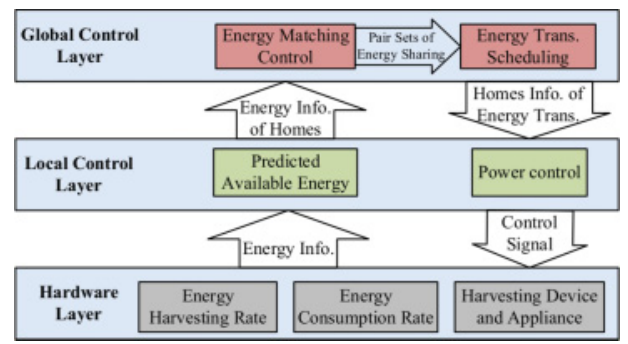

Fig. 3. Overview of system architecture.

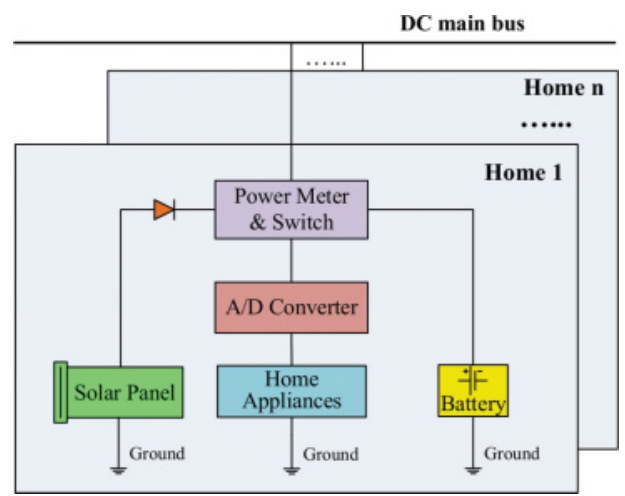

Fig. 4. Hardware architecture for energy sharing among multiple homes.

solar panels. Meanwhile, home appliances and batteries are bidirectionally connected to a power meter and a switch to measure and control the energy flow. The power meter and switch will be connected to the DC main bus for energy sharing with other homes.

- The local control layer will predict (i) renewable energy that a home expects to harvest based on weather forecast; (ii) expected energy consumption based on historical usage data. Then energy harvesting is compared against energy consumption for dividing homes into two categories. A home with energy surplus is called an energy supplier while a home with energy shortage is called an energy demander.

-The global control layer gathers information from homes to recommend home matchings which are most suited for energy sharing. Besides, it also determines the amount of energy for a home to share with other homes and to store in its local battery based on the current battery level and the expected available energy. Then, an energy transmission scheduling component will decide the order (schedule) of energy transmission based on matching results. The energy sharing algorithm used in our article is referred to as Global Energy Sharing (GES). Homes' information of energy sharing is transferred to a power controller in the local control layer, and finally, control signals are delivered to hardware for physical energy transmission.

\section{MODEL}

In this section, we describe the model used in our article. Let $X=\{1,2, \ldots, x\}$ be the set of homes. Time is divided into time slots. The size of a slot is referred to as window size $w$. Let $\triangle E_{i}(n w)$ be the difference between harvested energy $E H_{i}(n w)$ and consumed energy $E C_{i}(n w)$ for home $i$ in the time interval $[n w,(n+1) w]$. Homes are divided into energy suppliers, set $S$, and energy demanders, set $D$, according to whether $\triangle E_{i}(n w)$ is 
Table I. Notation of Parameters

\begin{tabular}{|c|l|}
\hline$E H_{i}(n w)$ & Harvested energy of $i$ in $[n w,(n+1) w]$ \\
\hline$E C_{i}(n w)$ & Consumption energy of $i$ in $[n w,(n+1) w]$ \\
\hline$\triangle E_{i}(n w)$ & $E H_{i}(n w)-E C_{i}(n w)$ \\
\hline$B_{i}(n w)$ & Energy level of battery deployed in $i$ at $n w$ \\
\hline$E_{i \rightarrow j}(n w)$ & Energy transferred from $i$ to $j$ in $[n w,(n+1) w]$ \\
\hline$\eta_{i \rightarrow j}$ & Energy transmission efficiency from $i$ to $j$ \\
\hline$E_{i}(n w)$ & Energy lack of $i$ after sharing in $[n w,(n+1) w]$ \\
\hline$\eta_{i}$ & Energy transmission efficiency of $i$ from AC line \\
\hline$r_{i}$ & Maximum transmission efficiency of $i$ \\
\hline
\end{tabular}

positive or negative. Note that it is possible that home $i$ has $\triangle E_{i}(n w)=0$. To simplify the notation, we also assign such homes into set $S$. Then, $S$ and $D$ partition $X$, i.e., $X=S \cup D$ and $S \cap D=\emptyset$.

When $i$ transmits $E_{i \rightarrow j}(n w)$ units of energy to $j$ via DC lines in time interval [nw, $n+$ 1) $w], j$ only receives a fraction of $E_{i \rightarrow j}(n w)$ due to energy loss. The energy loss during transmission contains both energy transmission loss over wires and battery conversion loss. Energy transmission loss over wires is mainly determined by the amount of energy transmitted, the length and type of power lines, and the transmission voltage. During the energy transmission, since renewable energy may not be consumed by a home immediately, extra energy might be stored in a local battery. The portion of energy stored in a battery during transmission introduces not only energy transmission loss but also battery conversion loss during battery charging. Thus, we should store a minimum amount of energy in a battery to reduce the energy loss.

Since the energy transmission takes time, the energy sharing should be based on the future energy information. For example, at time $w$, we make use of the energy information in time interval $[2 w, 3 w]$ to do energy sharing instead of energy information in time interval $[w, 2 w]$.

When a home still lacks energy after sharing, it fetches energy from the power grid via an AC line. This situation mainly occurs either at night when harvested energy is not sufficient or the transmission efficiency $\eta_{i \rightarrow j}$ between two homes is lower than transmission efficiency $\eta_{j}$ over the AC line.

Given supplier set $S$ and demander set $D$ over time, the purpose of energy sharing is to generate the ordered pair set $Q=\left\{\left(S_{1}, D_{1}\right), \ldots,\left(S_{m}, D_{m}\right), \ldots,\left(S_{|Q|}, D_{|Q|}\right)\right\}$ at time $n w$, where $S_{m} \subset S, D_{m} \subset D$ and $|Q|$ is the cardinality of $Q$. Our goal is to minimize the total amount of energy loss from battery, DC and AC lines:

$$
\sum_{n}\left(\sum_{i, j}\left(E_{i \rightarrow j}(n w) \cdot \eta_{i \rightarrow j}\right)+\sum_{i} E_{i}(n w) \cdot \eta_{i}\right)
$$

with the following constraint:

$$
\left|S_{m}\right|=1 \text { or }\left|D_{m}\right|=1, \quad m \in\{1, \ldots,|Q|\},
$$

where $i \in S ; j \in D ; R_{i}$ is the energy transmission efficiency of home $i$ from the AC line and $E_{i}(t)$ is the amount of energy shortage after the energy sharing. The constraint in Equation (2) ensures that there can be more than one supplier if they are providing energy to the same demander and more than one demanders if they are fetching energy from the same supplier.

An example of energy sharing is shown in Figure 5. With the bipartite graph of supplier set $S$ and demander set $D$, a matching result is given as home 1 provides energy to 2 ; 3 and 4 provide energy to 5 ; and, 7 provides energy to 6 and 8 . The energy 


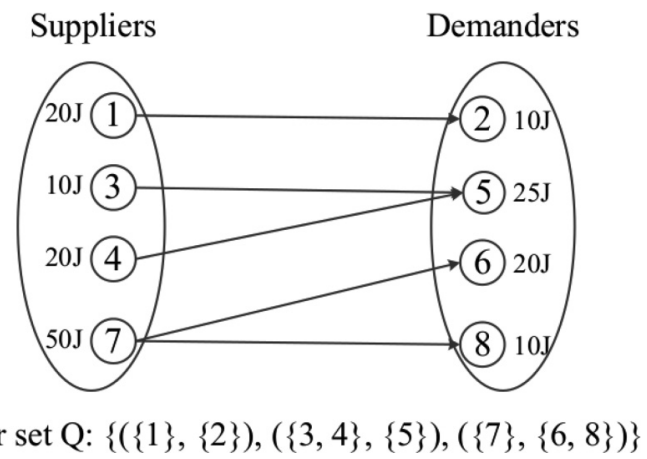

Fig. 5. An example of matching results $\left(S_{m}\right.$ and $D_{m}\left(\left\{S_{m}, D_{m}\right\} \in Q, m \in\{1, \ldots,|Q|\}\right)$ can be one home or multiple homes. The number in the $\bigcirc$ is the home id and the number outside the $\bigcirc$ is surplus or shortage energy).

transmission order follows the order of the pair set. The constraint in Equation (2) means we do not allow simultaneous energy sharing such as $(\{1,3\},\{2,5\})$. Otherwise, we cannot control whether energy transmission is from 1 to 2 and 3 to 5 or from 1 to 5 and 3 to 2 . The detailed discussion of energy transmission order is described in Section 5.5.

\section{SYSTEM DESIGN}

In this section, we introduce detailed system design for energy sharing. Firstly, we give energy harvesting and consumption prediction algorithms for predicting future energy information. Then, with the predicted energy information, we describe energy sharing within the cluster through matching energy among homes. We also explain how the energy sharing can be extended in homes with tree topology and propose a cluster selection algorithm to maximize transmission efficiency. Finally, we present a transmission protocol between homes and the cluster controller to assure that only one transmission executes in a cluster at one time.

\subsection{Renewable Energy Prediction}

For renewable energy, we use a prediction model similar to Sharma et al. [2010] that translates a weather forecast from the National Weather Service (NWS) into a solar or wind energy harvesting prediction. Since solar energy is the predominant renewable energy source in residential DG deployments, this article focuses on solar energy. However, the prediction model and energy sharing method can also be applied to other types of renewable energy such as wind energy. We briefly summarize the model below, which uses forecasted sky conditions to predict solar energy harvesting. The NWS releases a sky condition forecast, in addition to other weather metrics, every hour for the next 24 hours. At any time $t$, based on the sky condition percentage $C(t)$, we compute the solar panel's energy harvesting power $\mathrm{PH}_{i}(t)$ as:

$$
P H_{i}(t)=P_{\max } \cdot(1-C(t)),
$$

where $P_{\max }$ is the solar panel's maximum possible harvesting power. Sharma et al. [2010] quantify the accuracy of Equation (3) and show that it is more accurate than existing techniques that use the past to predict the future. Thus, based on Equation (3), at any time $t=n w$, we predict the solar energy harvesting within the next energy 
sharing interval $w$ as follows:

$$
\widehat{E H}_{i}((n+1) w)=\int_{n w}^{(n+1) w} P H_{i}(\tau) d \tau,
$$

where $w$ is the energy sharing window size.

\subsection{Energy Consumption Prediction}

To predict the home's energy consumption, we use a simple model based on an Exponentially Weighted Moving Average (EWMA). The EWMA exploits the diurnal nature of home consumption, and also adapts to seasonal variations. On a typical day, we expect the total energy consumption to be similar to the total energy consumption of previous days with slight deviations in weather and daily activities. More sophisticated models are possible that consider changing weekend activity patterns, weather conditions, or other information.

One goal of this work is to quantify how much renewable energy we are able to utilize with a simple and straightforward prediction model. Let $E C_{i}(n w)$ denote the amount of energy consumed in $[n w,(n+1) w]$ and $\widehat{E C}_{i}((n+1) w)$ denote the predicted energy consumed in $[(n+1) w,(n+2) w]$, which is given by:

$$
\widehat{E C}_{i}((n+1) w)=\alpha \cdot \widehat{E C}_{i}(n w)+(1-\alpha) \cdot E C_{i}(n w) .
$$

The value of $\alpha$ is chosen by using the method in Kansal et al. [2007]. Note that more sophisticated models that consider changing weekend activity patterns, weather conditions, or other data are possible. The energy harvesting and consumption prediction is not our main contribution and our framework is compatible with other consumption prediction models.

\subsection{Energy Matching Algorithm}

With the predicted energy harvesting and consumption information, we introduce the energy matching algorithm in this section. We first introduce the bipartite graph and then use a greedy energy search algorithm to match the energy among homes.

With the information of energy harvesting and consumption above, we generate the bipartite graph. If the harvested energy of home $i$ is larger than its consumed energy, $i$ is categorized to $S$, shown in the left set in Figure 5; otherwise, $i$ will be categorized to $D$, shown in the right set in Figure 5.

To maximize the overall transmission efficiency of the microgrid, we introduce the concept of maximum transmission efficiency of home $i$ as $r_{i}$. For home $i$, we calculate $r_{i}$ by matching energy with its neighbors in the descending order of transmission efficiency. When we fetch a new matching home, we update $r_{i}$ based on the amount of energy that can be shared between $i$ and $j$ and the energy loss rate between $i$ and $j$. The details of matching algorithms are shown in Algorithm 1. We first calculate maximum transmission efficiency $r_{i}$ at time $n w$ for every home (Lines 1-12). In Algorithm 1, we only give a description of $r_{i}$ for suppliers and note that $r_{i}$ of demanders can be calculated in a similar way. To calculate $r_{i}$, we first fetch the home $j$ with highest transmission efficiency with home $i$ (Line 4). Then, if home $i$ does not have enough energy for home $j$ (Line 5), home $i$ gives all the energy to home $j$ and the calculation of $r_{i}$ finishes (Line 6). Otherwise, home $i$ gives the amount of energy that home $j$ needs and continues the process for the calculation of $r_{i}$ (Line 9). After calculation of $r_{i}$, we fetch the home $i$ with highest maximum transmission efficiency (Line 13). If matching process of $i$ is not finished yet, we continue matching for $i$ (Line 14). If $i$ is a supplier, we find demander $j$ with highest transmission efficiency from $i$ (Lines 15 and 16). The amount of energy transferred from $i$ to $j$ is calculated with energy difference 


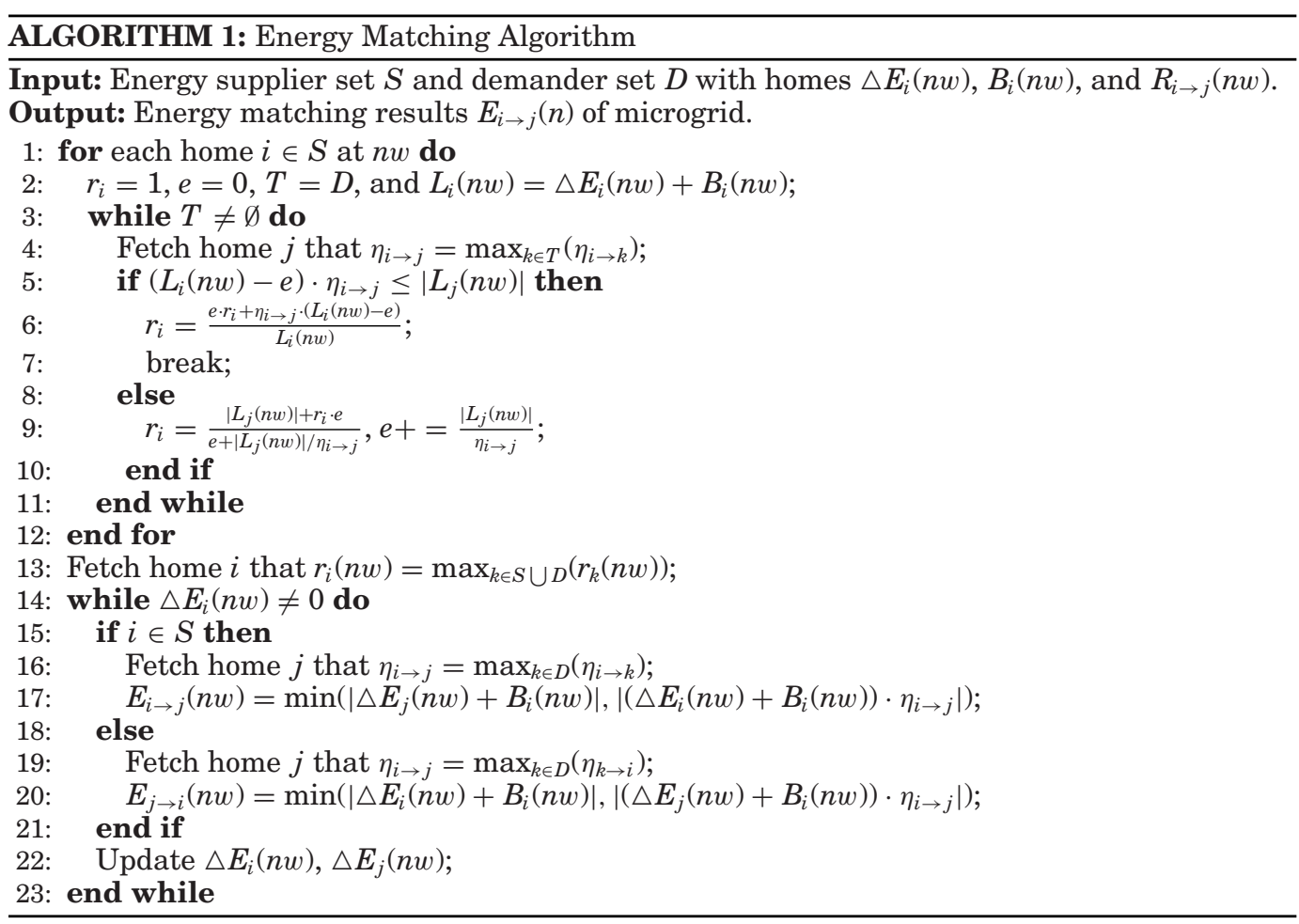

and battery level of two homes (Line 17). If $i$ is a demander, we find supplier $j$ with highest transmission efficiency to $j$, and the amount of energy transferred from $j$ to $i$ is calculated (Lines 18-21). We then update $\triangle E_{i}(n w)$ and $\triangle E_{j}(n w)$ (Lines 22 and 23).

Although Algorithm 1 does not guarantee the optimum solution of the energy matching problem, it has a low complexity. From Line 1 to 12 , the complexity is at most $|D| \cdot|S|$. From Line 12 to 22 , where we do the matching for suppliers and demanders, the complexity is also at most $|D| \cdot|S|$. Thus, the complexity of Algorithm 1 is $|D| \cdot|S|$. The optimal solution of the problem can be solved by minimizing transmission loss for every transmission combination. A transmission combination determines which demander will get energy from which supplier. The transmission combination with minimum transmission loss then can be the optimal solution. Here, we give a brief description of minimizing transmission loss for a specific transmission combination. For a specific transmission combination, we only need to decide the amount of energy a demander gets from those suppliers. Then, we can fetch the transmission pair with highest transmission efficiency and then assign the amount of transmission energy based on the energy difference and battery level of two homes. This process will continue until all transmission pairs are associated to a corresponding amount of energy. The complexity of the algorithm accounting for the matching followed by the energy assignment is $2^{|S| \cdot|D|} \cdot(|S| \cdot|D|)$. As the energy match interval is limited and the number of homes might be large, we adopt Algorithm 1 for energy matching instead of other sophisticated approaches.

\subsection{Energy Sharing Among Clusters}

In this article, we adopt the tree topology in Figure 2 to do energy sharing. This is because (i) the current power grid system uses a tree topology, which reduces the cost 
for reconstruction and design; (ii) since only one energy transmission can be executed at a time within the cluster, the number of homes in a cluster should be limited to reduce the energy transmission time. The reason why only one energy transmission can be executed at a time within the cluster will be introduced in Section 5.5. However, the energy matching algorithm we described above is mainly applicable for energy sharing within the clusters. In what follows, we extend to introduce how energy matching can be applied to a system with tree topology (see Algorithm 2).

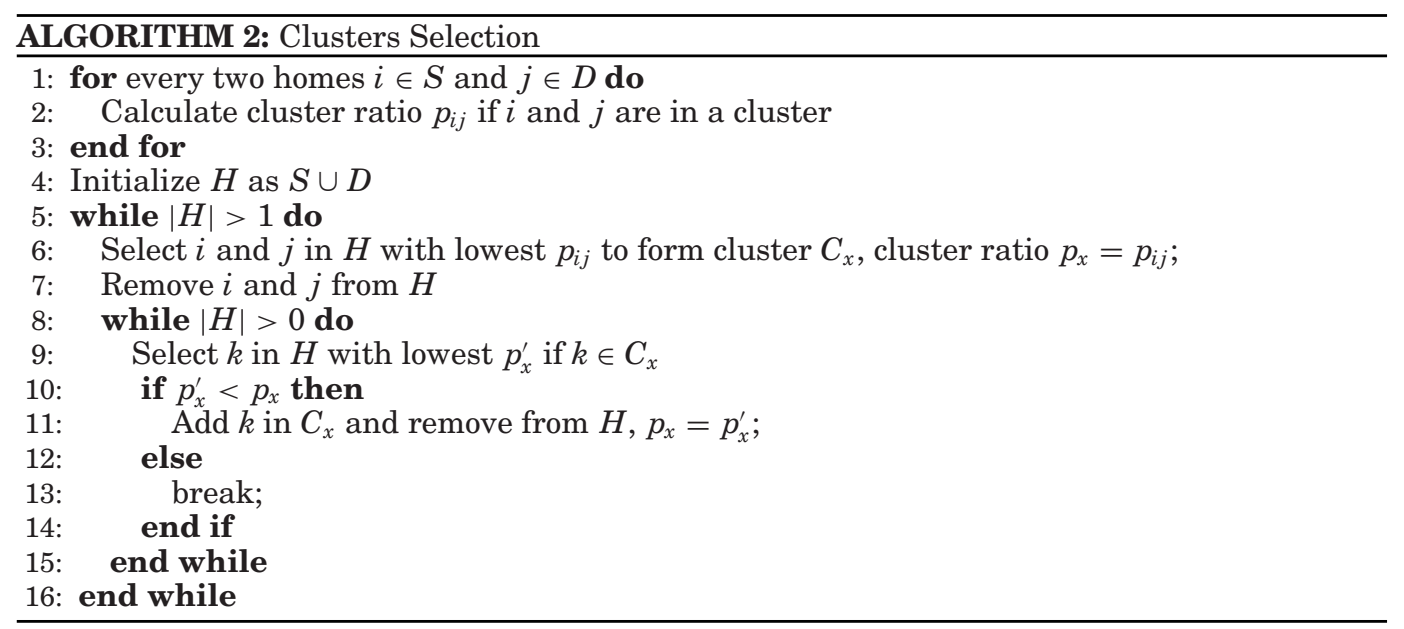

At the beginning, every cluster will execute Algorithm 1 for homes in that cluster with the energy information obtained from its children. The child can be either a home or a cluster of lower layer. The cluster checks if there is more than one child of itself free to execute the transmission. If yes, combine the transmissions in those children that can be executed simultaneously. A child is free if there is no transmission executing in the child.

However, we may have different transmission losses when considering different cluster selections. Since only one energy transmission can be executed at one time in a cluster, we should organize clusters with homes that have complementary energy consumption patterns to speed up the transmission process. A greedy algorithm is proposed to let most of the energy transmissions to be executed in parallel. The key idea is to select homes that their demand and supply match well to be in the same cluster. In our design, we propose a cluster ratio to evaluate whether the demand and supply of homes in a cluster match well. Cluster ratio of a cluster $C_{x}$ can be calculated as

$$
p_{x}=\frac{\left|\sum_{k}\left(\triangle E_{k}(n w)+B_{k}(n w)\right)\right|}{\sum_{k}\left|\triangle E_{k}(n w)+B_{k}(n w)\right|} \quad k \in C_{x},
$$

where $p_{x}$ is the cluster ratio of cluster $C_{x}$. For a cluster $C_{x}$ that only consists of two homes $i$ and $j, C_{x}=\{i, j\}$. In this case, we also use $C_{i j}$ to refer to such a cluster, then we also use $p_{i j}$ to refer to the cluster ratio of such a cluster. The details of the algorithm are described in Algorithm 2. We first calculate cluster ratio $p_{i j}$ for any two homes (Lines 1-3). Then we select two homes with the lowest $p_{i j}$ to form cluster $C_{x}$ and try to add other homes into the cluster (Lines 4-8). A new home $k$ will be selected with the lowest cluster ratio $p_{x}^{\prime}$ if $k \in C_{x}$ (Line 9 ). If cluster ratio $p_{x}$ decreases with $k$ added into 


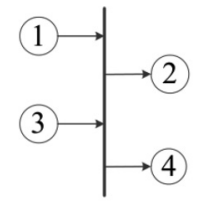

(a) Transmission chaos

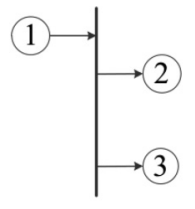

(b) Transmission scheduling

Fig. 6. An example of transmission chaos.

$C_{x}$, we add $k$ into $C_{x}$ and remove $k$ from $H$ (Lines 10 and 11). Otherwise, we try to form a new cluster until all homes are divided into clusters (Lines 12-16).

\subsection{Energy Transmission Protocol}

With the matching results, we can execute energy transmissions. The homes are all connected to one main bus of a DC line shown in Figure 6. If two energy transmissions $(\{1\},\{2\})$ and $(\{3\},\{4\})$ occur simultaneously, we cannot ensure the energy is transmitted from home 1 to 2 and 3 to 4 or home 1 to 4 and 3 to 2 , which we refer to as transmission chaos. A wrong matching may cause additional energy losses or billing problems. In fact, only transmissions with one supplier and multiple demanders or one demander and multiple suppliers do not cause transmission chaos.

To solve the problem, we develop a transmission protocol similar to Time Division Multiple Access (TDMA) to schedule energy transmission. The basic idea of transmission scheduling is to have transmissions executed simultaneously only if those transmissions do not cause transmission chaos. Fortunately, the matching results produced by Algorithm 1 are all one supplier with multiple demanders or one demander with multiple suppliers. Thus, we only need to let the cluster controller communicate with homes to make sure two transmissions do not execute at the same time.

The detailed communication protocol is shown in Protocol 3. For every controller, it first collects energy data and runs Algorithm 1 to get the matching results (Line 1). Then it checks if any demander or supplier sent the TRANS_END signal to the corresponding supplier or demander (Line 2). Then it checks if the transmission is executed as the matching results from Algorithm 1; if not, it re-runs Algorithm 1 with updated transmission details from the homes (Line 3). Note that the reason for rechecking stems for the fact that the energy data is sent to the controller is based on prediction; thus, there can be some prediction errors. It also needs to check if there are new children free to execute transmissions and add transmissions into a waiting queue $Q$ (Line 4). If no transmission is currently executing, it fetches the transmission with the highest transmission efficiency from $Q$ and sends TRANS_END signals to both demander and supplier (Line 5). The last thing for the controller is to make sure all the transmissions end before the next window, even if the transmission is not finished yet (Line 6). For every demander, it sends energy data to the cluster controller at a new window (Line 1). It then waits for TRANS_START signal to start the transmission (Line 2). If the demander receives enough energy, it sends TRANS_END to the cluster controller, ends the transmission, and sends back the transmission details (Line 3). All homes end transmission when they receive TRANS_END. However, receivers all need to send back transmission details when receiving TRANS_END because the transmission may not finish yet but may be terminated by the cluster controller (Line 4). The behaviors of the supplier are mostly similar to the demander, except it sends back TRANS_END when there is no more energy to transmit. The controller needs transmission details about how much energy is received at the demander. The TRANS_START signal should contain demander id, supplier id, and amount of energy for transmission, while TRANS_END 


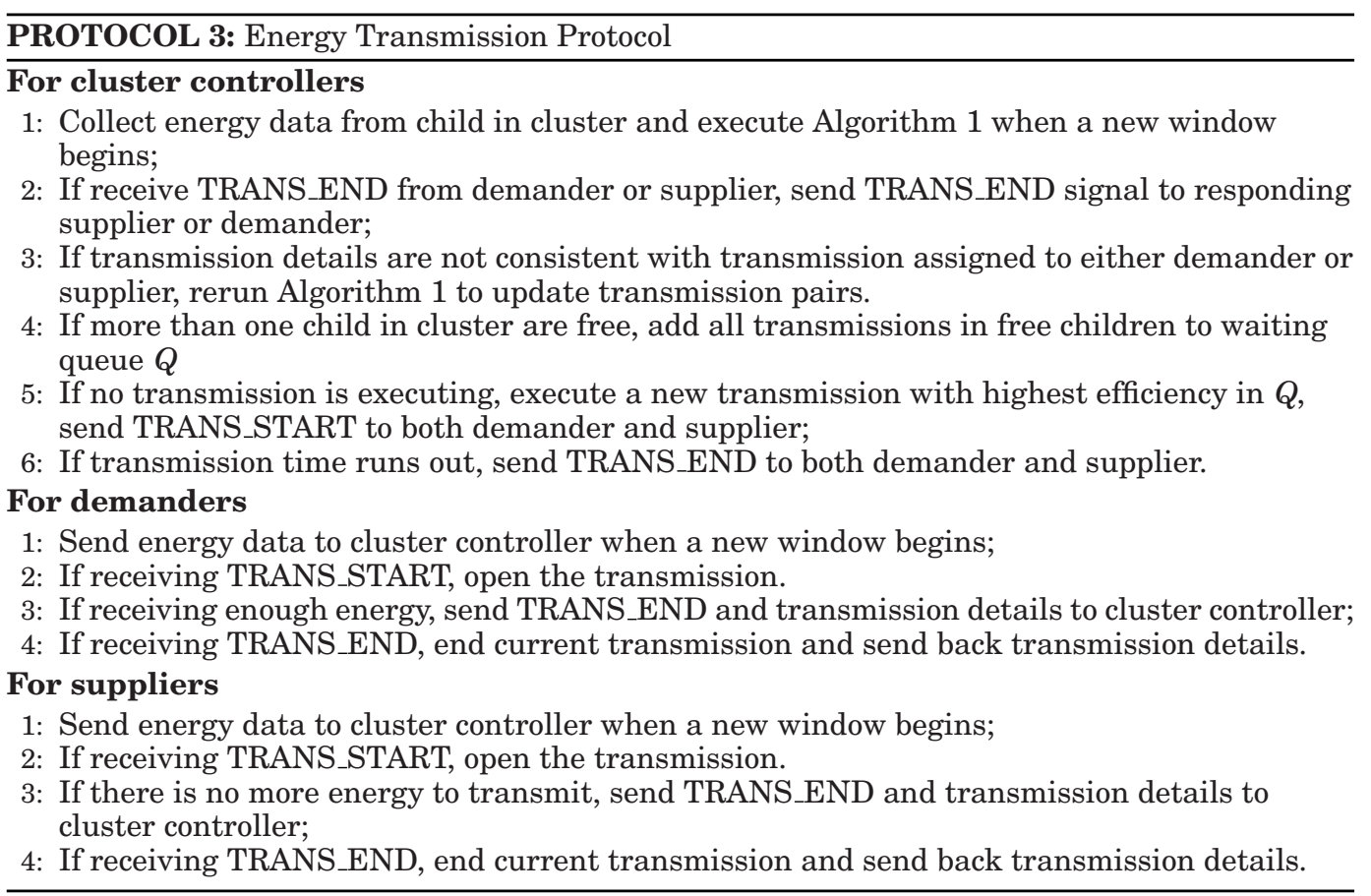

signal should contain demander id, supplier id, and amount of energy the demander receives.

\section{IMPLEMENTATION AND EVALUATION}

In this section, we evaluate the performance of our energy sharing system. We collect real data of energy harvesting and consumption in Amherst, MA; then, we compare the efficiency of our system against other solutions; finally, we show that our system can work with different battery sizes and home topologies.

\subsection{Data Collection}

We collect energy consumption data of 50 homes in Amherst, MA. We also deploy the solar panels in one home to collect the harvested energy data. The energy harvesting of a home is shown in Figure 7. In a day, the solar panel begins to harvest energy at around 7:00 a.m., reaches the energy peaks around 12:00 p.m., and ends the harvesting around 8:00 p.m. However, the amount of harvested energy in different days varies, which may be due to the varying weather conditions. Since the amount of harvested energy from solar panels is similar in a relative small area, we use the trace to produce harvested energy data of other homes with some randomness. The consumption data of homes consists of energy information collected every minute over 6 days. Harvested energy data is collected every hour. The weather forecast data we use is from the NWS (National Weather Station). Then, with the raw data, we calculate the prediction of harvesting and consumption data over 6 days and use it in our simulations.

\subsection{Evaluation Baselines and Metrics}

Baselines: To verify the efficiency of our energy sharing algorithm GES, we compare our design against (i) Oracle, which uses the same energy sharing algorithm as GES, but assumes real energy consumption and harvesting data for the next window is 


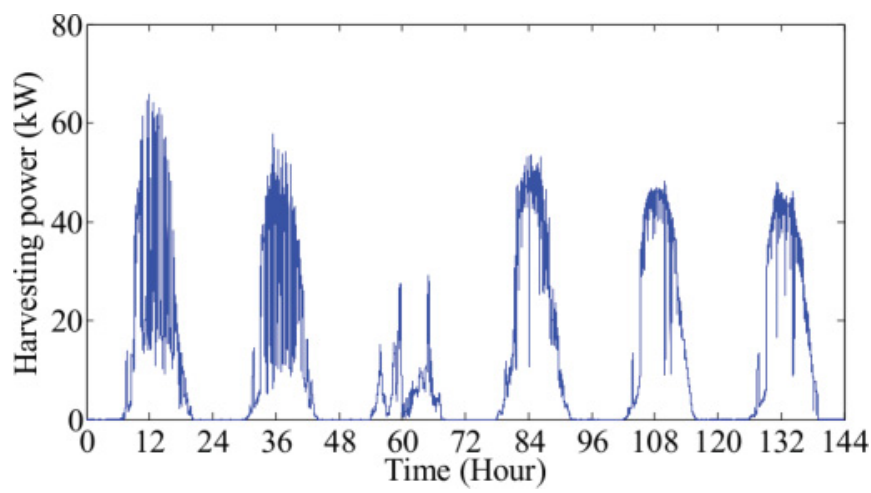

Fig. 7. Harvesting energy in 6 days.

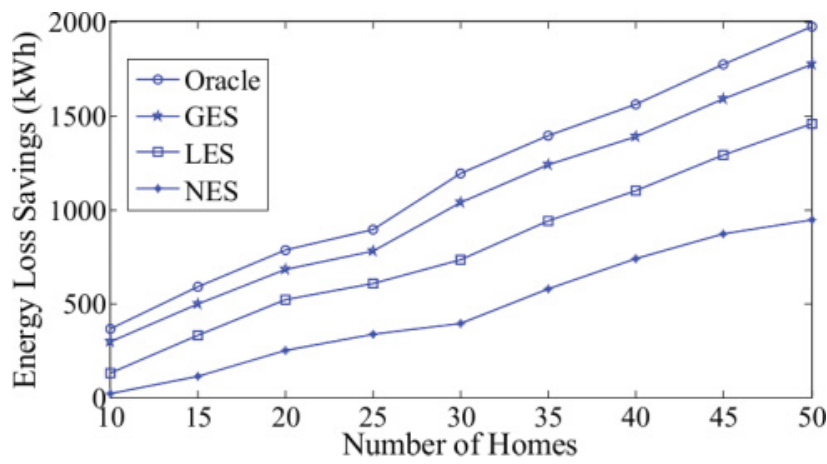

Fig. 8. Energy loss savings (total energy loss of four different algorithms, which includes transmission loss over wires, battery, and AC line).

available. We aim to show the impact of our prediction algorithm with the comparison; (ii) Local Energy Sharing (LES), which only allows homes to share energy with their nearest neighbor, thus the efficiency of energy sharing is low due to the stringent constraints for energy sharing; (iii) No Energy Sharing (NES), which adopts a large battery for every home to store all the surplus harvested energy for future usage; if there is no energy in the battery, it gets energy from the AC line.

Metrics: We use two metrics to evaluate the efficiency of our algorithm in our experiment; (i) Energy Loss Savings: energy loss compared to the strategy that homes always get energy from the AC line if there is no solar energy; (ii) Battery Size: the size of batteries that are deployed for homes to store extra energy.

\subsection{Basic Evaluation Results}

In this section, we will evaluate the effectiveness of our energy sharing system, which includes the energy loss savings and other detailed insights (detailed transmission loss, battery size distribution, and transmission over AC and DC lines) of our system. All results are simulated with the 6 days of empirical data of energy harvesting and consumption introduced in Section 6.1. The battery loss rate we use is $15 \%$ [Schoenung 2011]; AC and DC transmission loss rate is around $22.6 \%$ and $7.6 \%$, varying according to the transmission distance [Larruskain et al. 2005].

6.3.1. Energy Loss Savings. Figure 8 shows the energy loss savings of four different algorithms. In this simulation, the total number of homes varies from 10 to 50 and the 


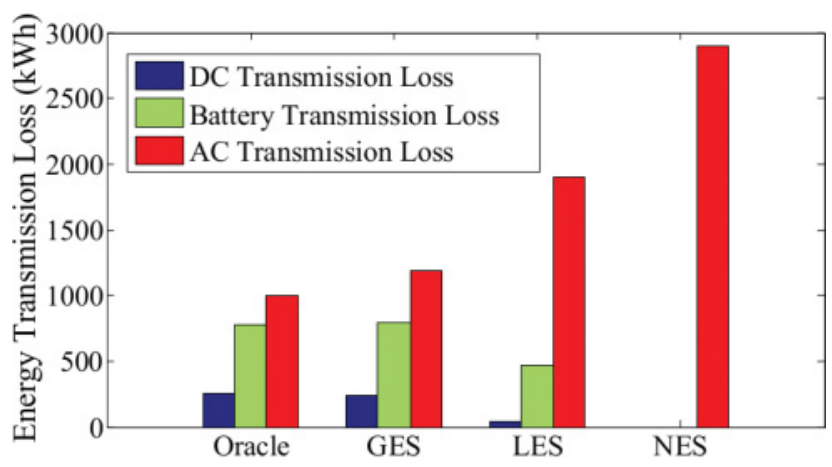

Fig. 9. Detailed transmission loss (transmission loss by DC wires, battery, and AC lines of 50 homes with 5 clusters).

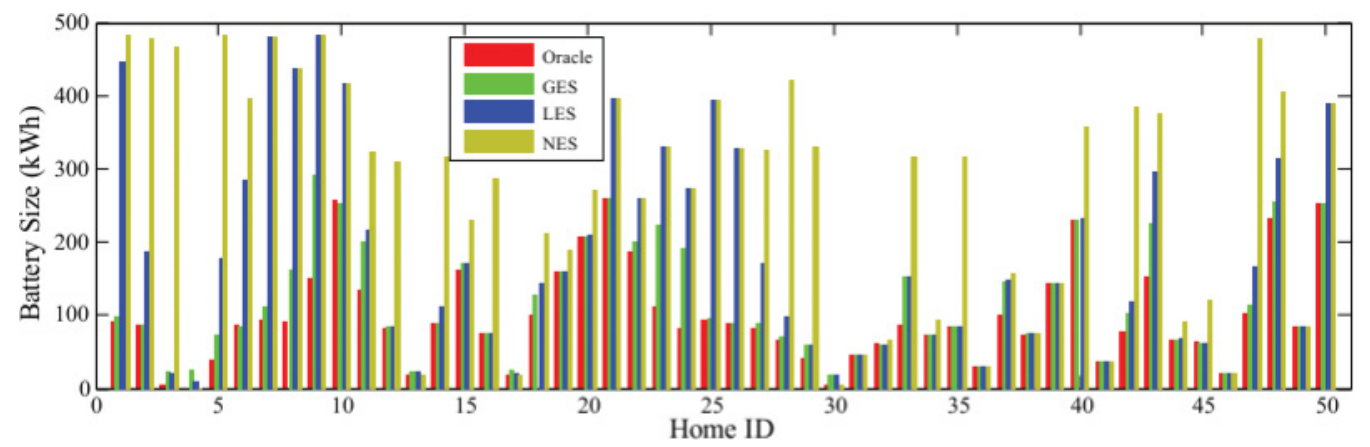

Fig. 10. Battery sizes needed for every home.

number of homes in one cluster is fixed as 10 (note that we do not use Algorithm 2 in this simulation; the performance of Algorithm 2 will be discussed in Section 6.4.2). In all four algorithms, the energy loss savings increase with the total number of homes because with more homes, more energy can be shared or stored in local batteries. Our algorithm outperforms LES and is very close to Oracle. NES performs the worst, since the harvesting and consumption in a home usually do not match; thus, many homes have less harvesting energy needed to get more energy from an AC line and the other homes have more harvesting energy that will waste their large batteries to store energy.

6.3.2. Detailed Transmission Loss. We also show the detailed transmission loss in Figure 9 . The result is for 50 homes with 5 clusters. The transmission loss over the AC line still occupies a large portion of the total energy loss. However, our algorithm reduces $60 \%$ AC transmission loss compared to NES. LES has less energy transmission over the DC line, therefore, it needs more energy from the AC line. Since the transmission loss over the $\mathrm{AC}$ line is larger than the battery conversion loss and DC transmission loss, the transmission loss of LES is larger, which explains Figure 8. Since the predicted data is not accurate, some homes may envision transferring more energy than they can harvest to other homes. In such cases, they have to get energy from the AC line after sharing with our algorithm. That explains why our algorithm needs more energy from both AC and DC lines compared to Oracle.

6.3.3. Battery Size. The battery size needed for every home is shown in Figure 10. Compared to NES, the other three algorithms need a much smaller battery size at 


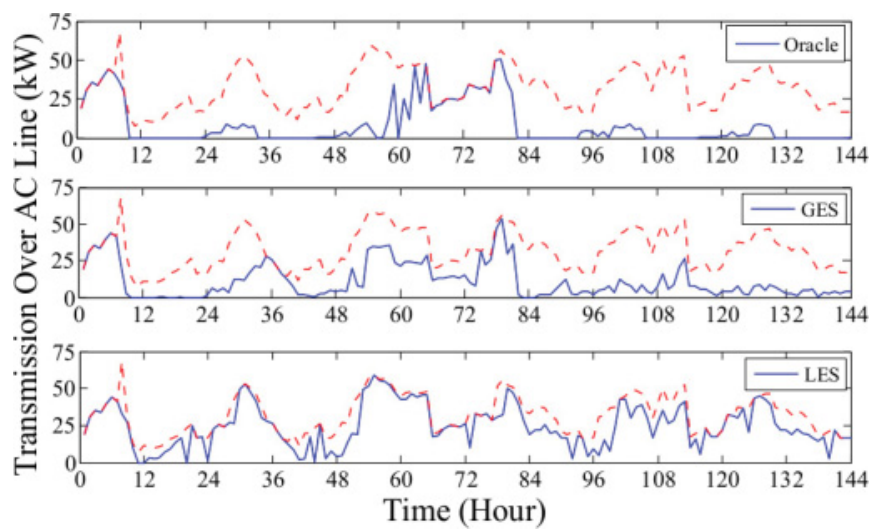

Fig. 11. Energy transmission over AC line (three algorithms (solid line) compared to NES (dashed line)).

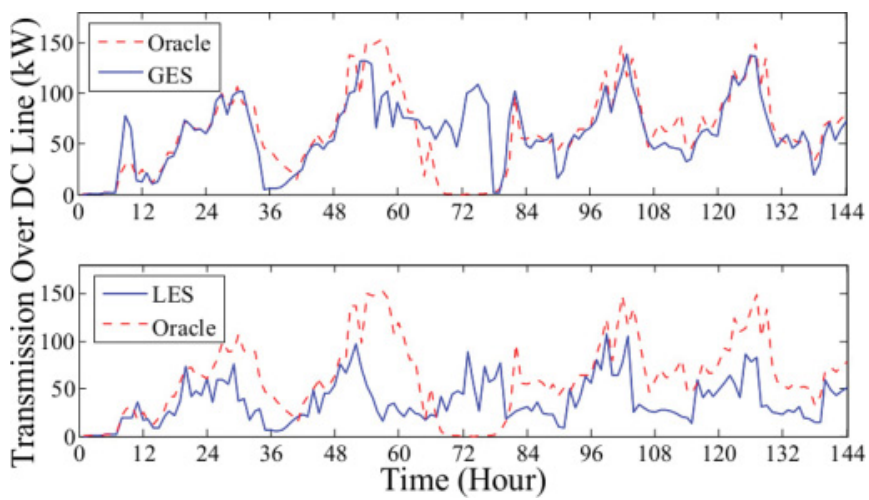

Fig. 12. Energy transmission over DC line (two algorithms (solid line) compared to Oracle (dashed line); NES does not use DC).

every home. The distribution of battery sizes among homes is proportional to the energy harvesting and consumption of homes. Our algorithm needs almost the same battery size as Oracle for every home and much less than LES.

6.3.4. Transmission Over $A C$ and $D C$ Line. The energy transmission over an AC line per hour is shown in Figure 11. All three energy sharing algorithms are compared to NES. For Oracle, homes seldom need any energy from an AC line except when harvesting energy from a solar panel is not enough in day 3 (hours 48 to 72 ). Our algorithm is very close to Oracle, in which, for nearly 10 hours of 1 day, homes do not need to get energy from an AC line. LES needs less energy than NES, especially when there is not enough harvesting energy at night. The peak of AC line transmission is also reduced from $70 \mathrm{KWh}$ to $50 \mathrm{KWh}$ in all three algorithms.

We show the energy transmission by DC per hour in Figure 12. The algorithms with less AC line transmission need more from DC, and NES does not have DC transmission. Note that in our algorithm, the prediction is not always accurate, thus, a home may envision transferring more energy than it will harvest in the next window. In such case, it needs to get energy from an AC line. Thus, even though energy transmission from an AC line of our algorithm is larger than one from Oracle, the DC transmission is still close to Oracle. 


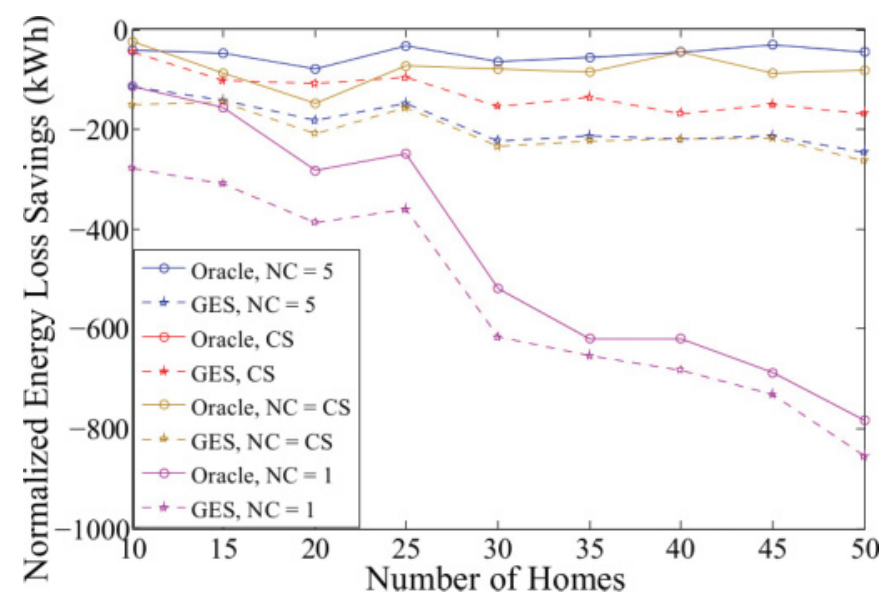

Fig. 13. Normalized energy loss savings of different cluster selections. CS: cluster selection algorithm introduced in Algorithm 2; NC: number of clusters.

\subsection{Advanced Evaluation Results}

In this section, we investigate the robustness of our system. We overview four main factors which affect performance of the system: (i) cluster selection; (ii) energy consumption pattern; (iii) battery size; and, (iv) energy prediction accuracy. The detailed setup for simulation is the same with the previous, if it is without specific description.

6.4.1. Impact of Cluster Selection. We show the impact of cluster selection to demonstrate the effectiveness of our system. Specifically, we use normalized energy loss savings to evaluate the performance of different algorithms, which is calculated by extracting energy loss savings of GES with Algorithm 2. Figure 13 shows the normalized energy loss savings of two different cluster selection algorithms. One is Algorithm 2 (CS) and the other is greedy selection. With a given number of clusters, greedy selection divides the microgrid into clusters with an equal number of homes based on distances between homes. Because the total number of homes is fixed as 50, with the increase of the number of homes per cluster, the number of clusters decreases. To show the performance of our system not only depends on the number of clusters in the microgrid but also detailed selection strategies, we also show the results of greedy selection with the same number of clusters as we get from Algorithm 2. Not surprisingly, the performance of the system improves with the increase in the number of clusters for greedy selection. This is because with more clusters, more energy transmissions can be executed in parallel, which reduces the amount of energy needed from the AC line. However, Algorithm 2 performs better than any greedy selections, even better than greedy selections with more clusters. This is because with Algorithm 2, homes with opposite energy consumption patterns are selected in the same cluster, which reduces the possibility for energy transmission over clusters, thus improves the performance of the system.

6.4.2. Impact of Energy Consumption Pattern. Since energy sharing is used to balance the mismatch of energy consumption and harvesting. The natural question is whether our system works when different homes in the microgrid have similar energy consumption patterns. In this section, we evaluate system performance under homes with similar energy consumption patterns. Energy differences of homes over time is shown in Figure 14. Each line in Figure 14 corresponds to energy consumption of a home. By visual inspection, we selected two sets of homes from our traces. In the top of Figure 14, 


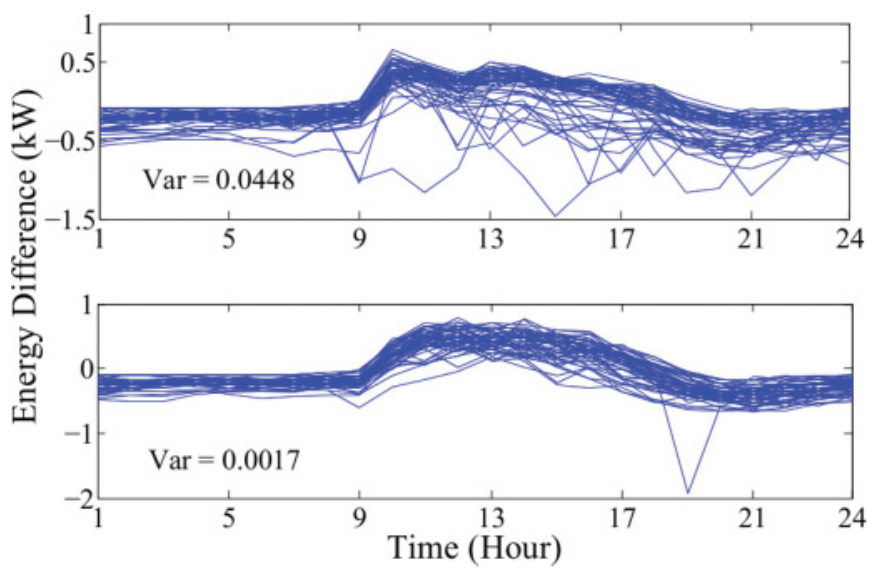

Fig. 14. Different energy consumption patterns.

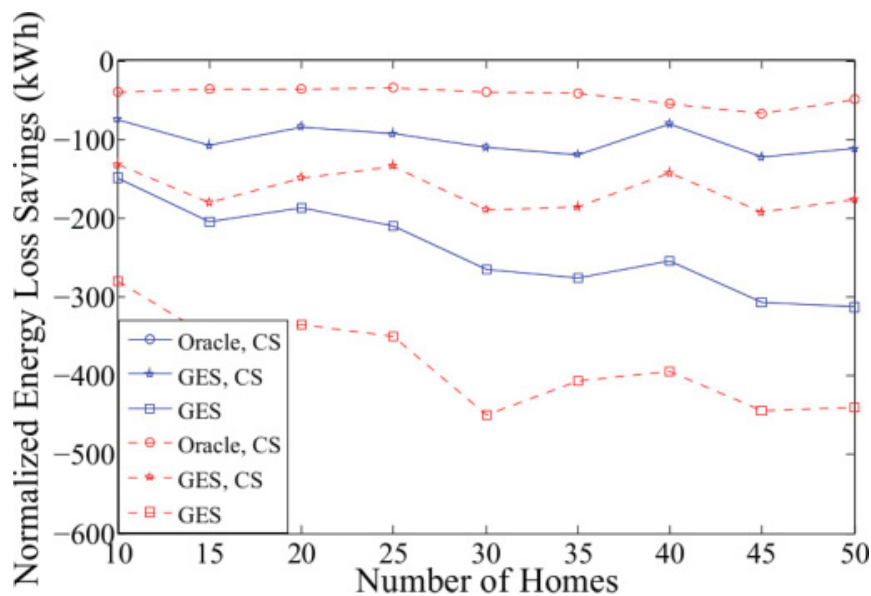

Fig. 15. Normalized energy loss savings of different energy consumption patterns. Blue solid lines are homes with different energy consumption patterns (Var $=0.0448$ ); red dashed lines are homes with similar energy consumption patterns ( $\operatorname{Var}=0.0017)$.

we selected a set of homes whose energy consumption pattern is as complementary as possible, i.e., favoring exchange opportunities. In the bottom of Figure 14, in contrast, we selected a set of users with similar consumption patterns, which does not favor exchanges. We refer to the users selected in the top (resp., bottom) of Figure 14 as microgrid 1 (microgrid 2, respectively). Note that the results obtained in the previous section considered the set of homes shown on the top of Figure 14. In general, both microgrids need energy in the early morning, have surplus energy at noon, and need energy in the evening. However, in microgrid 1, homes have different energy differences at noon while homes in microgrid 2 nearly have the same energy difference. The variances of energy differences of two microgrids are 0.0448 and 0.0017 , which also shows homes in microgrid 2 have more similar energy consumption patterns. Then, we repeat the simulations of the previous section and results are shown in Figure 15. With similar energy consumption patterns in microgrid 2, energy loss saving decreases for all algorithms. However, our system can still save $90 \%$ of energy loss compared to microgrid 1. Moreover, the gap between, with and without Algorithm 2 in microgrid 2, 


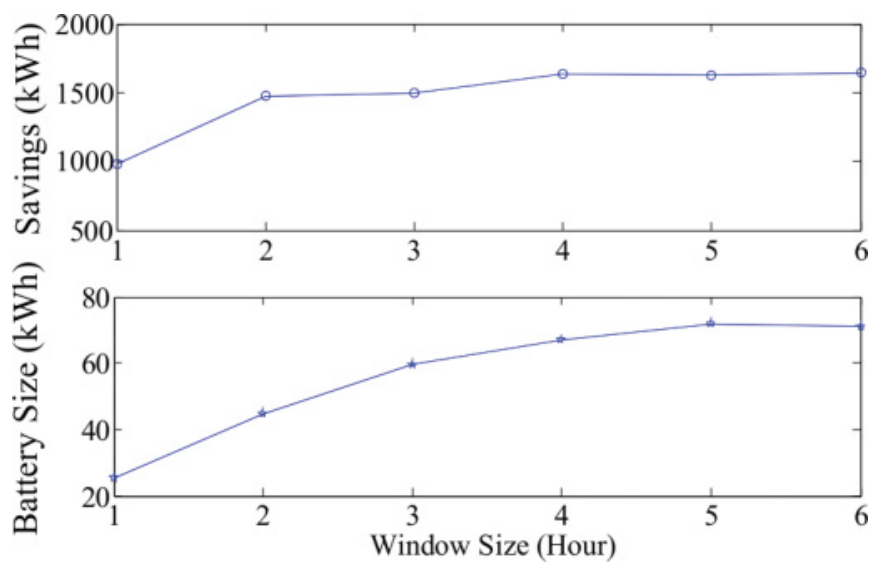

Fig. 16. Energy loss savings of different battery sizes.

is even bigger than the gap in microgrid 1, which means Algorithm 2 can even provide better performance improvement under homes with similar energy consumption patterns. This is because Algorithm 2 takes advantage of energy consumption patterns of homes; thus, when homes have similar consumption patterns, it can divide clusters more efficiently than the greedy selection algorithm.

6.4.3. Impact of Battery Size. As shown before, our algorithm can reduce the battery size needed by homes compared to NES. However, in some situations, the required large battery size is still not acceptable. In what follows, we consider constraints on battery size. We set the battery size to store energy that was harvested in two consecutive windows and show the amount of energy transmission loss savings with different window sizes in Figure 16. The result is using our algorithm for 50 homes with 5 clusters and 1 cluster. With the increase of the window size, the battery size increases for both 5 clusters and 1 cluster. And energy loss savings also generally increase with window size. The reasonable explanation is that with a large battery size, homes can use more energy from the battery but not the AC line. However, the increase rate decreases with the increase of window size. Thus, for different situations, the tradeoff should be balanced by the price of the battery and the energy loss savings.

6.4.4. Impact of Prediction Accuracy. Finally, we show the impact of prediction accuracy in this section. In our previous section, we make use of weather forecast and energy consumption history to predict future energy differences. However, prediction accuracy can vary under different environments. Thus, in this section, we evaluate our system with different prediction accuracy. Since it is difficult to attain prediction accuracy of different environments, we artificially generate prediction results with different prediction errors. And the prediction errors follow normal distribution. The detailed results are shown in Figure 17. The red stars are results with real trace data with $8.7 \%$ prediction error. We find that GES can save energy loss as long as the prediction accuracy is higher than 65\%, while LES and NES can only save energy loss when the prediction accuracy is higher than $71 \%$ and $75 \%$. This is because GES enables energy sharing between homes to alleviate prediction errors in a single home.

\section{DISCUSSION}

Return-on-Investment. In many instances, homes already have the necessary infrastructures to implement energy sharing. Many homes equip with solar panels and batteries to generate renewable energy. To implement energy sharing, the main 


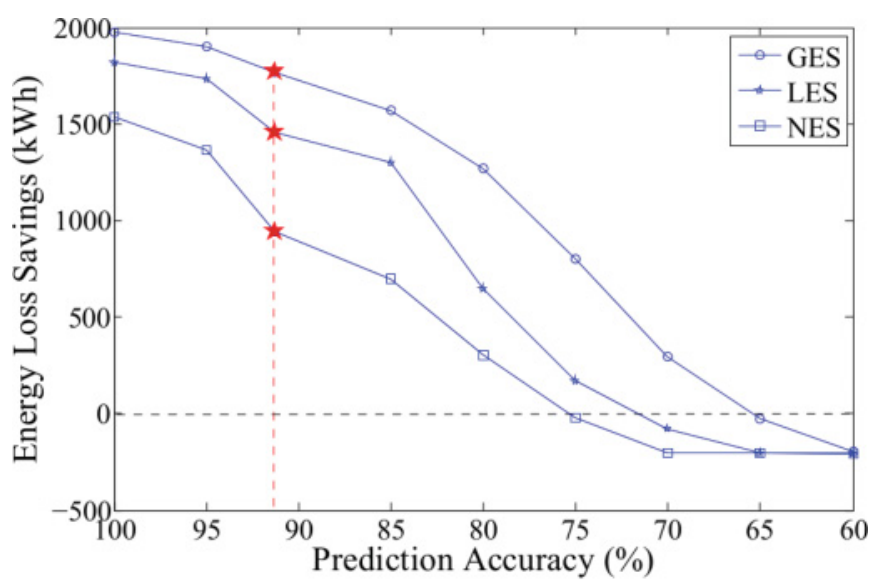

Fig. 17. Energy loss savings of different prediction accuracy.

Table II. Cost and Benefit for Different Window Sizes

\begin{tabular}{|c|c|c|c|c|c|c|}
\hline Window Size & 1 & 2 & 3 & 4 & 5 & 6 \\
\hline Cost $\left(\$ 10^{5}\right)$ & 2.55 & 4.50 & 5.97 & 6.70 & 7.22 & 7.12 \\
\hline Benefit $\left(\$ 10^{5} / y r\right)$ & 0.65 & 0.93 & 1.00 & 1.05 & 1.05 & 1.05 \\
\hline Years for Return & 3.88 & 4.84 & 5.97 & 6.34 & 6.88 & 6.77 \\
\hline
\end{tabular}

expense is to construct DC lines and larger batteries to store energy. For batteries, the price is around $\$ 200 / \mathrm{KWh}$, which can be cheaper in the future. For wires, the maximum power of a DC line is around 150KW; thus, we choose wires with $2 \mathrm{AWG}$ and the price is $\$ 14.58$ for 500 feet. Assuming the distance of 50 homes is 10 miles, then the total price for wires is $\$ 1,539$. The price of other equipment such as an inverter or energy monitor is listed in our earlier work [Mishra et al. 2012]. The benefit brought with our system design is mainly due to the savings of energy transmission over AC lines. The utility price in Amherst, MA is $\$ 0.13 /$ KWh. In Table II, we give an analysis of benefit and cost. The benefit and cost both increase with the window size. However, due to the significant increase ratio of battery size shown in Figure 16, the cost increases faster than the benefit. To return the investment sooner, a window size of 1 hour should be chosen for practicality.

Centralized vs. Distributed. Our current system design is a centralized control and a centralized cluster controller needs to collect energy harvesting and consumption information of all the homes in a cluster. However, since the number of homes in a cluster is limited, the computation and storage consumption would not be too high. After the energy sharing in a cluster, the cluster controllers only need to send the energy information of homes which still have energy surplus or shortage to a higher layer controller. Thus, the total computation and storage cost is under control. However, we also plan to develop distributed control in the future to allow homes to collect information from their neighbors for energy sharing to further reduce the computation and storage cost of controllers.

Sharing Price Design. Since we are mainly concerned about minimizing the energy loss in energy sharing, we do not pay much attention to the price of energy shared between homes. However, when considering the price factor, the incentives for homes to share energy may change. Some homes may not have enough incentives to share energy if the price of energy shared is low. However, we may consider to adapt some market-based solutions to design the price of shared energy in the future. 


\section{RELATED WORK}

Our research is related to energy efficient buildings, smart power grids, and workload scheduling.

- Research in energy efficient buildings mainly focus on energy auditing [Jiang et al. 2009] and design of control algorithms [Behl et al. 2011] to reduce energy consumption inside a single building. In addition, some of the work focuses on reducing the usage of building-wide heating, ventilation, and air-conditioning [Anil and Tomlin 2011]. Our work takes a different approach by investigating energy sharing among multiple nearby homes.

-In smart power grids, researchers have (i) developed models based on measurement from phaser measurement units to solve wide area control problems of large-scale power systems [Chakrabortty 2012; Chakrabortty and Salazar 2011; Chakrabortty et al. 2011], (ii) investigated the integration of renewable energy into power grids [Thatte and Xie 2012; Xie et al. 2012; Ilic et al. 2011a, 2011b], and (iii) optimized the packing size of large scale batteries to improve battery utilization in microgrids [Jin and Shin 2012]. Our work builds on previous works but targets on minimizing energy sharing loss over the small community level DC line.

- Since the price of electricity varies over time, related works focus on scheduling the workload to reduce the energy cost [Rao et al. 2010, 2012]. Rao et al. [2012] utilize both location and time diversity of electricity prices under multi-regional electricity markets to minimize the total electricity cost of data centers. Yao et al. [2012] takes a standard constrained model predictive control approach to smooth power demand and shave the power peak. Unlike previous works, our approach reduces the energy consumption without impacting the users' workload.

Even enormous work has been done and is still going on in the areas such as energyefficient building automation [Aswani et al. 2012], energy saving electronics [Crowcroft 2012; Vallina-Rodriguez et al. 2010], energy efficient data centers [Basmadjian et al. 2012; Mann et al. 2011], or optimal charging of plug-in hybrid electric vehicles [Sojoudi and Low 2011], but our work opens up a new approach where energy can be gained efficiently and used smartly.

\section{CONCLUSION}

In this article, we addressed the mismatch between harvested and consumed energy in individual homes by proposing energy sharing among nearby homes. We have (i) designed an efficient energy sharing system to share energy among homes, (ii) developed a lightweight energy matching algorithm and cluster selection algorithm to minimize the total energy transmission loss based on the predicted energy consumption and energy harvesting, and (iii) proposed an energy transmission protocol for controllers and homes to communicate through control signals to avoid transmission chaos.

We evaluated our system using empirical traces of harvested solar energy and home energy consumption in Amherst, MA. Through extensive simulations, we verified that our system (i) can reduce $64 \%$ of energy loss from an AC line by efficiently sharing energy among homes, (ii) can achieve high energy efficiency with low battery size, and (iii) is robust with different energy consumption patterns and energy prediction accuracy in a microgrid.

\section{REFERENCES}

A. Anil and C. Tomlin. 2011. Reducing transient and steady state electricity consumption in HVAC using learning-based model predictive control. Proceedings of IEEE 100, 1 (2011), 240-253.

A. Chakrabortty. 2012. Wide-area control of large power systems using dynamic clustering and TCSC-based redesigns. IEEE Transactions on Smart Grid 3, 3, 1503-1514. 
A. Chakrabortty and A. Salazar. 2011. Building a dynamic electro-mechanical model for the Pacific AC intertie using distributed synchrophasor measurements. European Transactions on Electric Power: Special Issue on PMU Applications. 21, 4, 1657-1672.

A. Chakrabortty, J. H. Chow, and A. Salazar. 2011. A measurement-based framework for dynamic equivalencing of power systems using wide-area phasor measurements. IEEE Transactions on Smart Grid 1, $2,68-81$.

A. Thatte and L. Xie. 2012. Towards a unified operational value index of energy storage in smart grid environment. 3, 3, 1418-1426.

A. Aswani, N. Master, J. Taneja, A. Krioukov, D. Culler, and C. Tomlin. 2012. Energy-efficient building HVAC control using hybrid system LBMPC. In Proceedings of IFAC Conference on Nonlinear Model Predictive Control.

R. Basmadjian, H. De Meer, R. Lent, and G. Giuliani. 2012. Cloud computing and its interest in saving energy: The use case of a private cloud. Journal of Cloud Computing: Advances, Systems and Applications 1, 5, 1-25.

M. Behl, M. Aneja, H. Jain, and R. Mangharam. 2011. EnRoute: An energy router for energy-efficient buildings. In IPSN.

J. Crowcroft. 2012. Cutting the energy cost of TV content distribution by 5, by understanding the popularity of the top ten programs. In e-Energy.

DOE. 2014. VEHICLE TECHNOLOGIES OFFICE: BATTERIES. Retrieved from http://www.energy.gov/ eere/transportation/vehicles.

DSIRE. 2010. Database of State Incentives for Renewables and Efficiency. (2010). http://www.dsireusa.org.

F. J. Jin and K. G. Shin. 2012. Pack sizing and reconfiguration for management of large-scale batteries. In Proceedings of International Conference on Cyber-Physical Systems.

Xiaofan Jiang, Minh Van Ly, Jay Taneja, Prabal Dutta, and David Culler. 2009. Experiences with a highfidelity wireless building energy auditing network. In SenSys.

Aman Kansal, Jason Hsu, Sadaf Zahedi, and Mani B. Srivastava. 2007. Power management in energy harvesting sensor networks. ACM Transction on Embedded Computer Systems 6, 4 (2007), 1539-9087.

L. Xie, Y. Gu, A. Eskandari, and M. Ehsani. 2012. Fast MPC-based coordination of wind power and battery energy storage systems. Journal of Energy Engineering 138, 2, 43-53.

D. M. Larruskain, I. Zamora, A. J. Mazn, O. Abarrategui, and J. Monasterio. 2005. Transmission and distribution networks: AC versus DC. In 9th Spanish-Portuguese Congress on Electrical Engineering.

Vijay Mann, Avinash Kumar, Partha Dutta, and Shivkumar Kalyanaraman. 2011. VMFlow: Leveraging VM mobility to reduce network power costs in data centers. In NETWORKING 2011. Springer, $198-211$.

M. D. Ilic, L. Xie, and J. Joo. 2011a. Efficient coordination of wind power and price-responsive demand Part I: Theoretical foundations. IEEE Transactions on Power Systems 26, 4, 1875-1884.

M. D. Ilic, L. Xie, and J. Joo. 2011b. Efficient coordination of wind power and price-responsive demand Part II: Case studies. IEEE Transactions on Power Systems 26, 4, 1885-1893.

A. Mishra, D. Irwin, P. Shenoy, J. Kurose, and T. Zhu. 2012. SmartCharge: Cutting the electricity bill in smart homes with energy storage. In e-Energy.

State of California. 2009. State of California Executive Order S-21-09. Retrieved from https://www.gov.ca.gov/ news.php?id=13269.

Lei Rao, Xue Liu, M. D. Ilic, and Jie Liu. 2012. Distributed coordination of internet data centers under multiregional electricity markets. Proceedings of the IEEE 100, 1 (2012), 269-282.

Lei Rao, Xue Liu, Le Xie, and Wenyu Liu. 2010. Minimizing electricity cost: Optimization of distributed internet data centers in a multi-electricity-market environment. In INFOCOM.

Lei Rao, Xue Liu, Le Xie, and Wenyu Liu. 2012. Coordinated energy cost management of distributed internet data centers in smart grid. IEEE Transactions on Smart Grid 3, 1 (2012), 50-58.

James Rose and Shaun Chapman. 2009. Freeing the Grid: Best and Worst Practices in State Net Metering Policies and Interconnection Procedures. Retrieved from http://www.newenergychoices.org/uploads/ FreeingTheGrid2009.pdf.

S. M. Schoenung. 2011. Energy storage systems cost update [A study for the DOE energy storage systems program]. Tech. Rep. SAND2011-2730, Sandia National Laboratories.

N. Sharma, J. Gummeson, D. Irwin, and P. Shenoy. 2010. Cloudy computing: Leveraging weather forecasts in energy harvesting sensor systems. In SECON.

S. Sojoudi and S. H. Low. 2011. Optimal charging of plug-in hybrid electric vehicles in smart grids. In Proceedings of the 2011 IEEE Power and Energy Society General Meeting. 
SERC: State Environmental Resource Center. 2011. ISSUE: NET METERING. Retrieved from http:// www.serconline.org/netmetering/stateactivity.html.

T. Zhu, A. Mishra, D. Irwin, N. Sharma, P. Shenoy, and D. Towsley. 2011. The case for efficient renewable energy management for smart homes. In ACM BuildSys.

Narseo Vallina-Rodriguez, Pan Hui, Jon Crowcroft, and Andrew Rice. 2010. Exhausting battery statistics: Understanding the energy demands on mobile handsets. In Proceedings of the 2nd ACM SIGCOMM Workshop on Networking, Systems, and Applications on Mobile Handhelds.

Jianguo Yao, Xue Liu, Wenbo He, and Ashikur Rahman. 2012. Dynamic control of electricity cost with power demand smoothing and peak shaving for distributed internet data centers. In ICDCS.

Received June 2015; revised February 2016; accepted April 2016 